

\title{
A NUMERICAL STUDY OF STELLARATOR TRANSPORT
}

\author{
W. N. G. Hitchon \\ Department of Electrical and Computer Engineering \\ University of Wisconsin \\ Madison, HI 53706 \\ and \\ H. E. Mynick \\ Princeton Plasma Physics Labaratory \\ Princeton University \\ Princeton, NJ 08544
}

\begin{abstract}
A detailed study of transport at low collision frequencies has been performed, using a numerical method of solution of the bounce-averaged fokkerPlanck equation which describes both ripple-trapped and non-ripple-trapped particles in a stellarator. Standard and "Transport-0ptimised" Stellarators are studied, the latter for the first time at collision frequencies which are low enough for the effects of a radial electric field to be important. Comparisons with Monte Carlo calculations show excellent agreement, and although existing analytic methods of solution can strictly only be applied in rather limited regions, some extensions of analytic results are discussed which enable us to explain quantitatively the behaviour observed.
\end{abstract}

\section{DISCLAIMER}

This report was prepared as an aecounl of work sponsored by an agency of the Unired States Government Neither the United States Goremment nor any agency thereof, nor any of their employees, makes any warranty, express or implied, or assumes any legal liability or responsibility for the accuracy, completeness, or usefulness of any information, apparatus, produch, or process disctoeed, or represents that its use would not infringe privately owned rights. Reference herein to any specific commercial product, process, or service by trade namen trademark, cnce herein to any speernic does not nectstarily constilute or imply its endorsement, recommenufacturer, or otherwise does United States Government or any agency thereor. The views and opinions of euthors expressed herein do not necessarily state or reflect thoee of the United Sintea Governaent or any agency thereof. 


\section{Introduction}

In this paper we present a detafled numerical study of what are expected to be the dominant transport processes in a large stellarator experiment or a stellarator reactor. First we consider "standard" stellarators and then "transport-optimised" stellarators. Care has been taken to describe correctly the distribution functions for ripple-trapped and for non-ripple-trapped particles and to match them onto each other at the trapping/detrapping boundary. A numerical approach is necessary because of the difficulty of obtaining analytic solutions which are continuous at this boundary. The status of the analytic theory is described below.

To introduce the issues involved, we consider the steps by which transport rates are calculated. Appraisal of transport at law collision frequency in non-axisymmetric tori involves the application of the ambipolarity condition. Using analytic expressions for the electran- and ionparticle fluxes one may determine the radiol electric field at which the fluxes are equal. Once this is known, the actual fluxes of particles and energy may be calculated $[1-3]$.

One complication arises because analytic expressions for the diffusion coefficients are local, and the finite excursions of particles from drift surfaces are not allowed for completely. At the highest energies this particular inadequacy of local diffusion coefficients becomes most pronounced. Apart from this, and the possibility that the radiat flunas will be affected by the flows which are induced on magnetic surfaces, for instance by the radial electric field, and various other physical processes which are neglected, there are two major problems with the expressions for the fluxes which are employed. 
The first problem involves the fact that, since the particles are distributed in energy, the fluxes have to be evaluated over a wide range of collision fraquencies corresponding to the different energies of the particles which are present. The problem here is that there is a lack of consensus as to the correct diffusion coefficients in some regimes.

Second?y, and more fundamentally, a difficulty arises when the transport rates are to be estimated at a collision frequency which is not unambiguously located in any of the usual 'regimes' but rather lies between two of them. These intermediace cases constitute regimes in themselves which are important not only because they are often as wide as the better-known regimes, but also because the (10w collision frequency) diffusion coefficient attains its largest value in one such regime.

A numerical method of solution of the appropriate Fokker-planck squation has been developed which avoids the last two problems. In reference [4] tive theoretical basis for the Fokker-Planck cade is described and the scaling of the diffusion coefficient $D$ with collision frequency studied for one device. In this paper, the scaling of $D$ with various parameters is studied in several collision frequency "regimes." The extension of the code to be appltcable to a more general class of devices is also described, having ripple-depths which vary with poloidal angle. The diffusion processes in these stellarators, which include "transport-optimi sed" stellarators, are then outlined.

The bounce-averaged Fokker-Planck equation for the distribution function is solved numerically on a two-dimensional grid, the two dimensions corresponding to poloidal angle $\theta$ and pitch-angle (the pitch-angle variable employed being nenoted as $y$. The solution is obtained by integrating the time-deperdent fokker-planck equation with respect to time. This method was selected because of its intuitive appeal, and because of the increased 
understanding of the solutions which is provided by being able to watch the evolution of the distribution function. The inefficiency of integrating over time is not a serious problem in the bounce-averaged scheme, espectally since most runs can be initiated using the distribution calculated for a similar data set. The time required to reach a steady state is drastically reduced as a result.

For $0 \leq y<1$, which is the ripple-trapped region, a single distribution function is obtained. However, for $1<y \leq y_{u}, y_{u}$ being the largest pitch angle allowed in the calculation, It is necessary to casculate the distribution functions for two classes of particles, having velocities parallel to the magnetic field which are in opposite directions.

The relationship between the three distribution functions is illustrated in Fig. 1. Ripple-trapped particles, whose distribution function is $f_{T}$, detrap at some point such as $A$ on the $y=1$ line and travel away from $y=1$ as toroidally blocked particles. Particles with $y>1$ which move in this direction form part of the 'forward' moving distribution, $f_{F}$.

Particles having $y>1$ which arrive at point $A$ moving in the opposite direction, being part of the 'backward' moving distribution, $f_{B}$, bounce at $A$ and join the forward distribution. At point 8 , also on the $y=1$ ine, particles in the forward distribution may entrap, with probability $P_{\text {tr }} \ll 1$, to join the trapped distribution $f_{T}$, or bounce, in which case they join the backward distribution, $f_{B}$. Other contributions to the transfer of particles from one state to another are provided by collisional scattering of particles. The calculated $f_{F}$ and $f_{B}$ describe all of the toraidally blocked particles, and some of the passing particles, for a sufficiently large choice of $y_{u}$. 
The trapping probability is determined by the Liouville condition from the velocities of the flows and the Jacobians immediately above and below $y=1$. This method is equivalent to the geometrical treatment of Goldston and Towner [5], but can be implemented in such a way as to conserve particle flux exactly, numerically. These' and other details of the flow velocities in the simple case where $\varepsilon_{h} \equiv \varepsilon_{h}(r)$, and the means by which flux js conserved precisely in the code, have been given previously [4]. Further discussion is postponed until Sec. 3, where stellarators with $\theta$-dependent ripple-profiles are introduced. Transport in such devices has not been studied previously at low collision frequencies.

One approximation to be noted is that a linearisation about a Maxiellian is perfarmed; an equation linear in the perturbed distribution function is obtained, with an effective source term $\dot{r} \frac{d F}{d r}, F_{m}$ being the Maxwellian. The most important effect of the radial motion is also retained in the "convected derivatives" of the perturbed part of $f$. This motion is not negligible and is especially important to describe the trapping process. If it is neglected, the orbits are of a quite different shape from the true trajectories.

By means of this numerical approach it is possible to solve for the distribution function in all of the regions of phase space which can contribute to transport (and not just $y<1$ ), for arbitrary collision frequency and without the questionable assumptions as to ordering of characteristic time scales or to boundary conditions which are often made in analytic theories. In this paper we shall examine the validity of existing results and show how they may be extended to bridge the more important gaps between the regimes discussed above, by means of numerical calculations. Although the fact that the code solves a bounce-averaged problem is in one sense its primary deficiency, it is also the case that the bounce-averaged 
formalation is most appropriate for comparison with analytic theorles. The latter are essential for the solution of the ambipolarity condition, as pointed out above, and in various other ctrcumstances.

The detailed information availab?e from the distribution functions ohtained in this way is not provired by Monte Carlo calculations, since purely guiding-centre calculations are prohibitively time consuming [6-10]. Even the faster bounce-averaged verstons of such codes [11] are able to provide an estimate of the diffusion coefficient but little useful information as to the distribution function in the more collisionless regimes. Monte Carlo calculations do, however, provide confirmation of the results obtained here for the diffusion coefficient, D, justifying the bounce-averaging procedure employed. This is somewhat surprising since the cases studied include some for which the ripple wells extend a significant distance poloidally, as seen by a particle moving along a field line. The shape of the well is not truly sinusoldal, and the particular bounce-averaging used would seem to be not entirely appropriate in these cases.

In the studies described below, the emphasis is put upon diffusion in the presence of a significani radial electric field, $E_{r} \gtrsim T / e a, T$ being the temperature, e the electronic charge, and a the minor radius. A radial electric field of this magnitude is an essential preoiction of transport theory in the stellarators considered, at low collision frequency. Virtually all of the particles which contribute significantly to transport in such a device will diffuse in a fashion corresponding to one or other of the regimes studied here. Only the most energetic particles (having a kinetic energy $k \geq T / \varepsilon, \varepsilon$ befing the finverse aspect ratio), will be capable of undergoing different diffusion processes than will the rest of the particles, since their motion is not dominated by the effects of the electric field to 
the same extent as is the motion of the other particles.

In what follows, the magnetic field strength is assumed to be of the form

$$
B=B_{o}\left[1-\varepsilon_{t} \cos \theta-\epsilon_{h}(r, \theta) \cos (e \theta+p \phi)\right],
$$

$\theta$ and $\phi$ being the poloidal and taroidal angles, respectively.

Section 2 is devoted to detailed studies of the transport in a "standard" stellarator. The basis for the calculation in such a device has already been given [4], along with some results for $D$ in a particular stellarator geometry. Section 3 describes the extension of the method of solution to the more general case where a $\theta$-dependent ripple is introduced, and discusses the performance of a transport-optimised stellarator at low collision frequency.

\section{Numerical Results for a Standard Stellarator}

In a previous paper [4], the overall shape of the $D$ versus $v / \Omega_{E}$ curve in the 'collision?ess' regime was examined and was shown to conform to theoretical expectations. $(D$ is the diffusion coefficient, $v$ the collision frequency, and $\Omega_{E}$ the $E \times B$ precession frequency.) In particular, regimes of low collision frequency transport were observed where 0 scaled as $v$ and $v^{1 / 2}$, with numerical coefficients which agreed remarkably well with the predictions of certain analytic theories $[12,13]$.

In this section we present results of a detailed study of low collision frequency transport. Analytic theories exist which predict three main regimes, the $v, v^{1 / 2}$, and $1 / v$ regimes, from the least collisional to the most. We begin with a comparison between, on the one hand, numerical results for the ' $v^{1 / 2}$ - regime' and its confluence with the ' $1 / v$ - regime' and, on the 
other, the boundary layer theory of Galeev and Sagdeev [12]. In this regime the results obtained agree well with analytic theory. According to that theory, transport is dominated, in a certaln range of collision frequencies, by the effects of a boundary layer of width $\Delta y_{b l} \sim \sqrt{\frac{V}{2 E_{h} \Omega_{E}}} \ll 1$ which forms immediately below $y=1$. We shali argue that the results of the boundary layer analysts for $f_{T}$ and $D$, when properly interpreted, apply even when $\Delta y_{b l} \sim 1$. For such values of $\Delta y_{b l}$ the scaling of 0 as $v^{1 / 2}$ saturates and may lead to a ' $\mathrm{plateau'}$ where $D$ is independent of . $v$. This result was suggested previously by Monte Carlo calculations [14]. Consideration of the distribution function provides a more convincing demonstration.

The ' $v$-regime' will be considered next. In this regime, the diffusion coefficient is less well explained by existing theory and is studied to test its scaling with parameters other than $v$, which has not been done previously. Figure 2 shows $D$ versus $v_{h} / \Omega_{E}$ for the device parameters 7 isted in Table I, which are used throughout, except as noted. $v_{h}$ is the effective collision frequency, $v / 2 \varepsilon_{h}$. The agreement between analytic theory [13] and the predictions of the code in the $v$ - reqime, which was extremely clase for the parameters employed in reference [4], is only within a factor of two for this device. A broad plateau is also evident, as mentioned above, as well as a $v^{1 / 2}$ regime on the $1 \mathrm{eft}$ and a $1 / v$ regime on the right.

The behaviour of $D$ at the highest collision frequencies studied, which should fall in the $1 / v$ regime, is discussed at the end of this section.

The scaling of $D$ with eilectric field strength in the low and intermediate collision frequency regines is mentioned in the appropriate subsections and again at the end of our discussion of the results for the "standard" stellarator. 


\subsection{Intermediate Collision Frequency}

Refore attempting the complicated task of demonstrating how $D$ scales with machine parameters in the $v$ regime and the $1 / v$ regime, we shall examine the intermediate range of collision frequency in some detail. The $v^{1 / 2}$ regime was studied analytically by Galeev and Sagdeev [12], but this reference contains several typographical errors so we shall first present the correct distribution function obtained from their analysis.

The "pitch-angle parameter" y is

$$
y=\left(\frac{\left(\kappa / \mu B_{0}-1\right)+\varepsilon_{t} \cos \theta}{2 \varepsilon_{h}}\right)+1 / 2
$$

where $k$ is the kinetic energy and $\mu$ is the magnetic moment.

In terms of $y$, the bounce-averaged Fokker-Planck equation employed by Galeev and Sagdeer is given in essence by:

$$
\Omega_{E} \frac{\partial f}{\partial \theta}+v_{d} \sin \theta \frac{d F_{m}}{d r}=\frac{\nu_{h}}{A^{2}} \frac{\partial}{\partial y} A \frac{\partial f}{\partial y}
$$

for collision frequencies $v_{h} \ll \Omega_{E^{*}} \quad v_{d}$ is the vertical drift velocity and $f_{T}$ has been expanded as $f_{T}=F_{m} ; \tilde{f}, F_{m}$ being the lowest order (Maxwellian) distribution function. Galeev and Sagdeev further $\operatorname{expand} F=X \cos \theta+Y \sin \theta$, and impose the boundary condtion $f(y=1)=0$. The numericial rusults tend to indicate that this boundary condition may lead to a solution which is approximately correct for these collision frequencies, al though no justification for it is givein and it leads to the wrong expresston for $D_{\nu}[c . f$. , Eqs. (5) and (6)] in the lower collision frequency regime unless $\tau_{\mathbf{r}} / \tau_{t} \ll 1$, which is untikely to be true. 
The asymptotic solution for $X$ and $Y$, valld $a y_{b l} \equiv \sqrt{v_{h} / \Gamma_{E}} \ll 1$, ( $v_{h}$ being in this case an effective col?isfon frequency at the centre of the boundary layer), is

$$
\begin{aligned}
& x=\frac{-v_{d} F_{m}^{\prime}}{\Omega_{E}}\left(1-\cos \left(\frac{1-y}{2 \Delta y_{b l}}\right) e^{-\left(\frac{1-y}{2 \Delta y_{b l}}\right)}\right) \\
& Y=\frac{v_{d} F_{m}^{\prime}}{\Omega_{E}} \sin \left(\frac{1-y}{2 \Delta y_{b l}}\right) e^{-\left(\frac{1-y}{2 \Delta y_{b l}}\right)}
\end{aligned}
$$

with $F_{m}^{\prime} \equiv \frac{d F_{m}}{d r}, r$ the minor radius.

To calculate the flux of particles or of heat, one takes $v_{d} \sin \theta i$ and integrates the appropriate moment of this quantity over polaidal angle, which eliminates the term involving $x$. Integrating next over pitch angle and omitting terms small in $\Delta y_{b e}$ yields a flux which scales as $\Delta y_{b e}$; a more careful integration by parts over pitch angle replaces this factor with one which rapidly saturates and then gradually decreases with increasing $4 y_{b e}$. This behaviour is reflected in the scaling of the diffusion coefficient in Fig. 2, that is, a fairly flat (or "plateau") region results. The agreement between numerical results and the analytic theory is excellent in the plateau as well as in the $v^{1 / 2}$ regime, although it is necessary to allow for che contribution of axisymmetric transport as well as ripple transport in the former case - see Fig. 2.

Fig. 3 compares the distribution function obtained from the code with that from analytic tieory, for a collision frequency such that $\Delta y_{b e} \ll 1$ and for two cases having $\Delta y_{b e} \sim 1$. The agreement below $y=1$ is good in al 1 cases, although the theory is based on the assumption that $\Delta y_{b l} \ll 1$. The results of integrating the analytic form for the distribution function over the region shown in these figures yields a diffuston coeficient 
whose value is denoted as $\mathrm{D}_{v} 1 / 2$ and $\mathrm{D}_{v^{0}}$ in $\mathrm{Fig} .2$. In a wide region between the $v$-regime and the $1 / v$ regime, the agreement between the "analytic result" and the numerical result is very close indeed, for these parameters. (0f course, the axisymmetric contribution to transport must be allowed for when it becomes comparable in magnitude with the ripple transport.) The scaling as $v^{1 / 2}$ results from the increase in the number of particles involved in the transport as $\Delta y_{b l} \sim v^{1 / 2}$ grows. The saturation of $D$ occurs as $\Delta y_{b l}$ approaches unity and the "boundary layer" fills the ripple-trapped region, $y<1$, in the . "plateau" region mentioned above.

It is shown in Ref, 14 that the scaling of $D$ with $\left|E_{1}\right|$ should be as $E_{r}-3 / 2$ when $\Delta y_{b \ell} \ll I$ and $E_{r}^{-1}$ when $\Delta J_{b \ell} \sim 1$. As discussed at the end of this section, and as shown in Fig. 6, the numerical results agree very closely with the predicted sca! ings in these cases.

In the $\nu^{1 / 2}$ regime, the diffusion coefficient is expected to be independent of $\varepsilon_{h}$. Fig. 4 shows the variation of $D$ with $\varepsilon_{h}$, in this case $D$ is approximately constant, except for very small values of $\varepsilon_{h}$ when $v_{h}$ is large and the diffusion coefficient is no longer given by the Galeev-Sagdeev result. The code results for the smallest $\varepsilon_{h}$ actually show an increase in $D$, by a factor of about 2. As $\varepsilon_{h}$ is decreased, D must eventually be dominated by the transport arising at $y>1$, and in fact the bulk of the radial transport in this case (about $75 \%$ ) occurs where $y>1$. This is to be contrasted with the negligible total fluxes expected (and observed) above $y=1$ in this, the $v^{1 / 2}$ regime for more typical values of $\varepsilon_{h}$. In the $v^{1 / 2}$ and plateau regimes we have found a clear interpretation of our results in our 'extension' of Galeev and Sagdeev's theory [12]. It is also encouraging to note that guiding-centre and hybrid bounce-averaged/guiding-centre results [15] agree very closely with the present code in this and all other regimes. 


\subsection{Low Collision Frequency.}

We now return to consideration of the $v$ regime.

In order to introduce the zerminology necessary for discission of the $v$ regime, we consider the collisionless particle motion again. Referring again to Fig. 1, we define $\tau_{r}$ to be the time spent drifting from the trapping point (B) to the detrapping point (A) whilst the particle is ripple-trapped. When the particle detraps (ai $A$ ), it travels to point $B$ in a time $\tau_{b t} / 2, \tau_{b t}$ being the time for one complete toroidal bounce. Since the trapping probability, $P_{F T}$, is small, the particle is most likely to bounce, returning to $A$, where it must bounce, and then to $B$ again.

Before it traps, the particle will bounce $N$ times at or near $B$, where on average $N \cong 1 / P_{F T}$. The total time spent toroidally blocked is $\tau_{t}=(N+1 / 2) \tau_{b t} \approx N \tau_{b t}$. The ratio $\tau_{r} / \tau_{t}$ is formally of order unity in the parameter $\mathrm{m} / \mathrm{e}$, but in Ref. 13 as elsewhere it was assumed that this ratio is large compared to unity. For the parameters given in Table I, this ratio is not large. When $\tau_{r} / \tau_{t} \gg 1$, the diffusion coefficient in the $v$ - regime is found to be

$$
D_{v}=\frac{v\left(E_{h} / 2\right)^{1 / 2}}{\varepsilon_{t}}\left(\frac{v_{d}}{\Omega_{E}}\right)^{2}
$$

In the opposite limit, $\tau_{r} / \tau_{t} \ll 1$, it was found from Mante Carlo calculations [15] that a reasonable fit to th data was given by

$$
D_{v}=v\left(e_{h} / 2\right)^{-1 / 2}\left(\frac{v^{v}}{s_{C}}\right)^{2}
$$

Here, $v_{d}=\frac{m v_{1}^{2}}{2 q B R}, \Omega_{E}=\frac{E_{r}}{r B}$, and $\varepsilon_{h}=\varepsilon_{h o} r^{\ell}$, other symbols having their usual meanings. 
There is an apparent discrepancy between analytical and numerical results, in that Fig. 2 exhibits a $v$ regime which lies a factor of two below the value given by Eq. (5). However, Yushmanov [16] has pointed out that the tivo diffusion coefficients shouid be added reciprocally. Since $\varepsilon_{h} / \varepsilon_{t} \approx 1$, the two results are roughily equal and so combining them in this way would produce the observed result: a reduction by a factor of two.

Whichever of Eqs. (5) and (6) applies, the scaiting with $\left|E_{r}\right|$ is expecter to be as $E_{r} \cdot 2$, and this appears to agree very well with the resuits shown in Fig. 6.

$D_{\nu}$ versus $\varepsilon_{h}$ is shown in Fig. 5. Since too large a variation in parameters wouid move the plasma into the $v^{1 / 2}$ regime, only a few points are shown.

The variation of $D$ with $\varepsilon_{h}$ is expected to be as $\varepsilon_{h}^{1 / 2}$ when $\frac{\tau_{r}}{\tau_{t}} \gg 1$, while according to Eq. (6), the variation is as $\varepsilon_{h}^{-1 / 2}$ in the opposite limit. The time spent ripple trapped is $\tau_{r} \sim 1 / \Omega_{E}$. The time spent toroidally trdpped is given by $\tau_{t}=\tau_{b t} / P_{F T}$, whilst $P_{F T}$ is given approximately by [4] (aithough the $\theta$-dependence is clearly not accurate):

$$
P_{F T}=\frac{\pi^{2}}{\sqrt{2}} \frac{\Omega_{E}^{R}}{\hat{u p}_{\mathrm{H}}} \cot \theta
$$

and

$$
\hat{u}=2\left(\frac{E_{h}{ }^{\mu B}}{M}\right)^{1 / 2} .
$$

The time for a complete toraidal bounce, $\tau_{b t}$, is of the order of $\frac{\pi R}{1 w_{1}}$. The rotational transform in an $\ell=2$ stellarator is $t=\varepsilon_{h o}^{2} k^{4} / p^{3}$, where 
$\varepsilon_{h}=\varepsilon_{h o} r^{2},[17]$. Since $v_{1} \sim\left(\frac{r}{R} \frac{\mu B_{0}}{M}\right)^{1 / 2}$ for a torotdally trapped particle,

$$
\tau_{b t} \sim \frac{\pi p^{3}}{R^{5 / 2} \varepsilon_{h o}^{2}}\left(\frac{M}{\mu B{ }_{0} r}\right)^{1 / 2} .
$$

Consequently,

$$
t_{t} \sim r^{1 / 2} p^{4} \tan \theta /\left(R^{7 / 2} E_{h o}^{3 / 2} \Omega_{E} v_{\perp}^{2}\right)
$$

For small values of $\varepsilon_{h o}$, $\tau_{t}$ may exceed $\tau_{r}$, principally because the rotational transform decreases and so the connection length becomes large. Thus when $\varepsilon_{h}$ is small, the scaling with $\varepsilon_{h}{ }^{1 / 2}$ given by Eq. (5) goes over to a variation as $\varepsilon_{h}^{-1 / 2}$, according to Eq. (6). At though the precise slope is not clear from Fig. 5, some of the points in the intermediate range of values of $\varepsilon_{h}$, show a slope of $-1 / 2$, and a distinct minimum in $D$ is observed in the same region, as expected:

\subsection{Higher Collision Frequency}

As indicated in Ref. 4, the $v^{-1}$ regime is expected to be superimposed on the "banana diffusion" which also arises in an axisymetric tokamak. The calculated diffusion coefficient falls very slowly from the plateau which we discussed in connection with the saturati in of the collisional boundary layer, then eventually begins to drop as $v$ increases, but with a coefficient which is an order of magnitude bigger than the predicted $\nu^{-1}$ - regime coefficient. These results appear to be consistent with the ripple transport being partially masked by the onset of banana - then axisymmetific plateau diffuston. The sum of the "helical" and the axisymetric contributions to 
diffusion is shown in Fig. 2, and agrees very closely with the numerical results for $\sigma=0$,

To test the validity of the results of the present calculation, Monte Carlo simulations were performed using a hybrid bounce-averaged/guiding-centre code, in which the upper limit in $y$ at which radial motion of particles is permitted, $y_{m}$, may be varied. In this way the effect of including transport due to the motion of particles in banana orbits may be studied. For a case where $y_{m}$ is only slightly greater than one, the "standard" 1/v regime was recovered [15]. The significance of this result is that, when the radial motion of particles in toroldally blocked orbits (that is, "tokamak bananas') was suppressed, the expected stellarator transport was obtained.

When the Monte Carls calculation allows for the full guiding-centre drifts up to $y=2$, that is, it includes radial motion of tokamak bananas, then in the saine, $v^{-1}$, regime, the agreement between Monte Carlo simulation and the Fokker-Planck code is again excellent. It seems that the behaviour observed, as described above, is indeed confirmed by the Monte Carlo calculation. The usual theory of ripple transport in this regime [12] uses an inappropriate boundary condition at $y=1$. As we have pointed out elsewhere, the distribution function is not a Maxwe 1 ian above $y=1$, in part because of radial motion in tokamak banana orbits, and it appears that as a consequance it is rarely correct to assume that the perturbed part of the trapped particle distribution function goes to zero at $y=1$.

Finally in this section, we remark that Fig. 6 can be construed as evidence for the existence of the distinct regimes of transport to which we refer. The $D$ versus $\nu_{n} / \Omega_{E}$ curve is less convincing since the results can be regarded as a single smooth curve with no clear transitions. By choosing arbitrary points within the supposed regimes and showing that $D$ behaves quite 
differently at each of them with respect to changes in $E_{r}$, a distinction which agrees with the analytic theories we have discussed - can be made. Note that by changing $E_{r}$, we are changing $v_{h} / \Omega_{E}$, even though $v$ is constant in these comparisons. As a result, we are comparing points which are spread throughout the regimes they represent, and so it is not mereily coincidence that we have found a value of $v$ at which $D$ scales with $E_{r}$ in the expected way. Rather, it seems to scale this way throughout at leasi a portion of each regime.

\section{Transport-Enhanced Stellarators}

Stellarators in which the ripple amplitude depends on the poloidal angle, some of which show dramatically improved transport properties in the $1 / v$ regime, have been studied in that regime by several authors $[2,18-20]$. The restriction to the $1 / v$ regime is the result of the inaccessibility of the lower collision frequency regimes to study by means of guiding-centre Monte Carlo calculations, and t're difficulty of performing an analytic calculation of the distribution at low $\nu$ when $\varepsilon_{h} \equiv \varepsilon_{h}(r, \theta)$. This constraint is largely removed by the development of a hybrid bourice-averaged/guiding-centre approach which is much faster than a purely guiding-centre code [15]. Studies of a variety of such novel stellarator configurations at low collisionality, using this method, will be reported elsewhere. Here we describe the modifications to a previous numerical calculation of the distribution function [4] which are necessary to allow for a poloidatiy varying amplitude of the "ripple," $\varepsilon_{h} \equiv \varepsilon_{h}(r, \theta)$, and the results of the extended calculation. 


$$
\varepsilon_{h}=\hat{\varepsilon}_{h}(r)(1-\sigma \cos \theta)
$$

$\sigma$ is a constant whilst $\hat{\varepsilon}_{h} \sim r^{\ell}$. The drift velocities obtained for this form of $\varepsilon_{h}$ were

$$
\begin{aligned}
& \dot{r}=v_{d} \sin \theta\left(1-\frac{\dot{\varepsilon}_{h}}{\varepsilon_{t}} \overline{\cos n}\right), \\
& \dot{r}=v_{d}\left(\cos \theta+\frac{l \varepsilon_{h}}{\varepsilon_{t}} \overline{\cos n}\right),
\end{aligned}
$$

which are the result of using the form of the helical ripple (15) in the expression (14) and setting $\Omega_{E}=0$.

Diffusion in this particular model field will be studied in some detail in what follows. First we mention one other model magnetic field of interest, however. In Ref. 21 it was shown that the effective helical ripple amplitude in a simple stellarator is modified from the usual form, $\varepsilon_{h}=\varepsilon_{h o} r^{2}$ by the gyration of the field iine. When the field strength is expressed in $f 1 u x$ coordinates, so that variations in $B$ along a field lirie are made apparent, we find that it is necessary to modify $\varepsilon_{h}$;

$$
\varepsilon_{h}=\varepsilon_{h o} r^{l-1} z, \quad z=\left(r^{2}-2 r z_{0} \cos \theta+z_{o}^{2}\right)^{1 / 2},
$$

where $z_{0}=\ell R / p^{2}$ and $p$ is the number of field periods in the toroidal direction. $z$ is the distance of the field point from a point which is a distance $z_{0}$ major radially inward from the magnetic axis. (These definitions differ from those used in Ref. 21, where $z$ and $z_{0}$ were normalised to the minor radius.) 
In this case, the drift velocities become

$$
\begin{aligned}
& \dot{r}=v_{d} \sin \theta\left(1+\frac{\varepsilon_{h o}}{\varepsilon_{t}} r^{\ell} \frac{z}{z} \overline{\cos n}\right) \\
& r \dot{\theta}=r \Omega_{E}+v_{d}\left(\cos \theta+\frac{\varepsilon_{h o} r^{\ell}}{\varepsilon_{t}}\left[(l-1) \frac{z}{r}+\left(\frac{r-z a-\operatorname{se}}{z}\right)\right] \overline{\cos n}\right)
\end{aligned}
$$

In the limit $z_{0}+0$ the helical ripple in the magnetic field becomes $\varepsilon_{h}=\varepsilon_{h o} r^{2}$, as is usually assumed, since $z+r$. In this case, the "helical" term in $\dot{r}$ vanishes, but in $r \dot{\theta}$ a term $v_{d} l \varepsilon_{h} / \varepsilon_{t} \overline{\cos n}$ remains, from the corresoonding term. The usual neglect of this term is justified since $r^{\pi_{E}} \gg v_{d}$ and this term does not contribute to the divergence if $z_{0}=$ ?.

The code employed actually solves for the distribution function on a twodimensional mesh in $(y, \theta)$ - coordinates, with $r$ reduced to the status of a parameter. In order to avoid a nonphysical distortion of the distribution function when the kiretic equation solved by the code is written in conservation form [4], we must ensure additionally that the two-dimensional flow is divergence-free:

$$
\frac{1}{J_{2}}\left\{\frac{\partial}{\partial y}\left(J_{2} \dot{y}\right)+\frac{\partial}{\partial \theta}\left(J_{2} \dot{\theta}\right)\right\}=0
$$

We achieve this by adring an extra, small term $\Delta \dot{\theta}$ to $\dot{\theta}$, effectively absorbing $\frac{\partial}{\partial r}\left(J_{2} \dot{r}\right)$ into $\frac{\partial}{\partial \theta}\left(J_{2} \dot{\theta}\right)$. Let $\dot{\theta}_{t}$ denote the "true" rate of change of the paloidal angle, given by Eq. (14). The "effective" rate of change, $\dot{\theta}_{\mathrm{e}}$, is

$$
\dot{\theta}_{\mathbf{e}}=\Delta \dot{\theta}+\dot{\theta}_{t} \quad \text {. }
$$


Where $\Delta \dot{\theta} \ll \dot{\theta}_{t}$. $\dot{y}$ employed in the code has its true value since $\dot{\theta}$ used in Eq. (13) is $\dot{\theta}_{t}$.

The madification to $\dot{\theta}$, whilst maintaining the divergence of the velocity appearing in the Equation being solved equal to zero, introduces an extra term $\Delta \dot{\theta} \partial f / \partial \theta$ into the Fokker-Planck equation which is, however, negligible [4]. The term we dropped from the Fokker-Planck equation in omitting $i \frac{\partial f}{\partial r}$ is also genuinely sma11, since the derivative is at constant $y$. Had any other pitch-angle variable been employed, this would not necessarily be true.

The change in $\dot{\theta}$ is given by

$$
\Delta \dot{\theta}=-v_{d}\left[\frac{1}{\varepsilon_{t}} \frac{\partial \varepsilon_{h}}{\partial r} \frac{1}{\cos n}+\frac{1}{\varepsilon_{h}} 1 / 2_{r} \frac{\partial}{\partial r}\left(r / \varepsilon_{h}^{1 / 2} d(\cos \theta)\right)\right] \text {. }
$$

The first term exactiy cancels one term in $\dot{\theta}_{t}$. If $E_{h}$ is independent of $\theta$, the second term is readily simplified, yielding $-2 v_{d} \frac{\cos \theta}{r}$ for $\ell=2$. Otherwise, we assume $\varepsilon_{h}=(a+b \cos \theta)^{\alpha}$, in which case this term is

$$
\frac{-v_{d}}{r \varepsilon_{h}^{1 / 2}} \frac{\partial}{\partial r}\left(\frac{r}{b} \frac{\varepsilon_{h}^{(\alpha+2) / 2 \alpha}}{(\alpha / 2+1)}\right)
$$

The two properties of the equation being solved that we have discussed so far, namely, that the equation be written in conservation form and (in order to achleve this) that the velocity have zero divergence, must also be realised in the numerical implementation of the equation. If the numerical schema does not embody these properties, then even though the analytic expressions are correct, errors will accumulate during the run which significantly distart the solution. 
The velocities employed in the present work are such that the finite differencing in $\theta$ places a requirement on the $y$-velocity, if the numerical divergence is to be zero. The analytic expression for $y$, for an $\ell=2$ "sigmaconfiguration" is, from Eq. (12),

$$
\dot{y}=\frac{-A}{A}\left[\frac{v_{d} \sin \theta}{r}+\left(\Omega_{E}+\frac{v_{d} \cos \theta}{r}\right) \frac{\sigma \sin \theta}{2(1-\sigma \cos \theta)}\right]
$$

The expression which is actually employed, which reduces.to Eq. (23) as the mesh size $\Delta \theta$ goes to zero, is

$$
\dot{y}=\frac{-A}{A}\left[\frac{v_{d}}{3 r} D_{1}(\theta)+\left(\Omega_{E}+\frac{4 v_{d}}{3 r \sigma}\right) D_{2}(\theta)\right],
$$

where

$$
\begin{aligned}
& D_{1}(\theta)=\left(c^{-} \sqrt{1-\sigma c^{-}}-c^{+} \sqrt{1-\sigma c^{+}}\right) /\left(2 \Delta \theta \sqrt{1-\sigma c^{0}}\right), \\
& D_{2}(\theta)=\left(\sqrt{1-\sigma c^{+}}-\sqrt{1-\sigma c^{-}}\right)\left(2 \Delta \theta \sqrt{1-\sigma c^{0}}\right),
\end{aligned}
$$

and $c^{+}=\cos (\theta+\Delta \theta), c^{0}=\cos \theta, c^{-}=\cos (\theta-\Delta \theta)$.

Equation (24) is designed to cancel exactly the results of the numerical calculation of the contribution of the poloidal motion to the divergence. Similar considerations determine the precise numerical form of $\dot{\theta}$ employed in the code.

Provided the divergence of the phase-space velocity is zero, the FokkerPlanck equation can be written in conservation form. Precise numerical conservation of particles is ensured in the code since the exact fluxes which are subtracted at one mesh point are added two mesh spaces away in the appropriate directions. 
We now consider the velocities in the other region of phase space, corresponding to non-ripple-trapped particles $(y>1)$. The expression for $\dot{r}$ given in Eq. (14) is also valid for $y>1$. Since $\dot{r}$ is assumed to he negligible in calculating $\dot{y}$ above $y=1$ (but not in the source term $\dot{r} \frac{d F}{d r}$ ), the only relevant analytic divergence criterion in this region is identical to Eq. (19). Provided we use $\dot{y}=\dot{\theta} \frac{d y}{d \theta}$, and the same expression for $\dot{\theta}$ as given in Ref. 4 , then the analytic divergence condition may be shown to be satisfied for $\varepsilon_{h} \equiv \varepsilon_{h}(r, \theta)$. The numerical equivalent of the analytic divergence condition is then easily satisfied, using expressions for the velocities derived in the same way as Eqs. (24) and $(25)$.

b) The Trapping Probabitity

In order to ensure that the flux of particles is conserved at the $y=1$ boundary, the trapping probability must be calculated to be consistent with the flow velocities on either side of $y=1$.

To establish the appropriate expression for the trapping probability, $P_{F T}(\theta)$, we introduce $\gamma_{T}^{\theta}$, the flux in the $\theta$-direction, and $Y_{T}^{y}$, the $y-f 1 u x$, where the subscript $\tau$ refers to one of the three distributions, $\tau=T$ (trapped), $F$ (forward), or B (backward). Then $r_{T}^{\theta}=J_{2} \dot{\theta}_{T} f_{T}$ and $r_{T}^{y}=J_{2} \dot{y}_{T} f_{T}$, and $J_{2}$ is derived for the various regions in Ref. 4. We also use $\hat{r}_{\tau}^{\theta} \equiv J_{2} \dot{\theta}_{\tau}$, etc.

The numerical form of the derivative with respect to $\theta$ is denoted $D_{\theta}$. We impose the conditions which determine $P_{F T}$, namely, $f_{T}=1$ be a steady-state solucion of the kinetic equation; the equations in question may be written as, first, for the forward distribution, 


$$
0=D_{\theta} \hat{r}_{F}^{\theta}\left(\theta, 1+\frac{\Delta y}{2}\right)+\left[\hat{r}_{T}^{y}\left(\theta, 1-\frac{\Delta y}{2}\right)-\hat{\gamma}_{B}^{y}\left(\theta, 1+\frac{\Delta y}{2}\right)-\hat{Y}_{F}^{y}\left(\theta, 1+\frac{3 \Delta y}{2}\right)\right] / 2 \Delta y
$$

at the mesh point fmmediately above $y=1$, where $y=1+\frac{\Delta y}{2}$. At this point the outgoing ilux consists of two parts, the flux which goes into the trapped region, $\left\{\hat{r}_{T}^{y}\left(\theta, 1-\frac{\Delta y}{2}\right)\right]$, and the reflected flux, $\left[-\hat{r}_{B}^{y}\left(\theta, 1+\frac{\Delta y}{2}\right)\right]$. This latter term is equal to $\left[r_{B} r_{F}^{y}\left(\theta, 1+\frac{\Delta y}{2}\right)\right]$, where $r_{B}$ is the $r^{\prime}$ io of the hackward to the forward flux when $f_{\tau}=1$.

At the same point the equation for the backward distribution yields

$$
0=D_{\theta} \dot{\gamma}_{B}^{\theta}\left(\theta, 1+\frac{\Delta y}{2}\right)+\left[-P_{F B} \dot{r}_{F}^{y}\left(\theta, 1+\frac{\Delta y}{2}\right)-\dot{r}_{B}^{y}\left(\theta, 1+\frac{3 \Delta y}{2}\right)\right] / 2 \Delta y .
$$

The important point here is that part of the forward flux at $y=1+\frac{\Delta y}{2}$, which is reflected at $y=1$, becomes the incoming flux in the equation for the backward distribution, also at $y=1+\frac{\Delta y}{2} \cdot P_{F B}$ is the fraction reflected; $P_{F T}$ is the fraction transmitted, that is, the trapping probability, so $P_{F T}=$ 1 - $P_{F B}$, which ensures conservation of particles.

$$
\begin{aligned}
& \text { Similarly, then, at } y=1-\frac{\Delta y}{2}, \\
& 0=D_{\theta} \hat{r}_{T}^{\theta}\left(\theta, 1-\frac{\Delta y}{2}\right)+\left[r_{T}^{y}\left(\theta, 1-\frac{3 \Delta y}{2}\right)-P_{F T} \hat{r}_{F}^{y}\left(\theta, 1-\frac{\Delta y}{2}\right)\right] / 2 \Delta y .
\end{aligned}
$$

The quantities $P_{F T}$ and $r_{B}$ are obtained from these equations, rather than from more usual anatytic expressions [5], in order to ensure that not only is flux conserved but also that Liouville's theorem is satisfied exactly at $y=$ 1. Using $\gamma_{B}^{y}=r_{B} r_{F}^{y}$ in Eqs. $(26)-(28)$, we find conditions that determine $P_{F T}$ and $r_{B}$ :

$$
P_{F T}(\theta)=\frac{2 \Delta y 0_{\theta} \hat{\gamma}_{T}^{\theta}\left(\theta, 1-\frac{\Delta y}{2}\right)+\hat{\gamma}_{T}^{y}\left(\theta, 1-\frac{3 \Delta y}{2}\right)}{\hat{\gamma}_{F}^{y}\left(\theta, 1+\frac{\Delta y}{2}\right)}
$$


and $r_{B}$ is given by

$$
\frac{P_{F B}-r_{B}^{2}}{r_{B}}=\frac{r_{T}^{y}\left(\theta, 1-\frac{\Delta y}{2}\right)}{r_{F}^{y}\left(\theta, 1+\frac{\Delta y}{2}\right)} \text {. }
$$

Finally, $\dot{\gamma}_{F}^{\theta}\left(\theta, 1+\frac{\Delta y}{2}\right)$ is determined by "integrating" Eq. (26), which introduces a small correction to the "analytic" expression at $y=1+\frac{\Delta y}{2}$.

c) The Geometry of the Mesh.

The transport-optimised configurations studied by Mynick et al., [18] differ from a standard stellarator in that the ripple is (to some extent) localized at either $\theta=0$ or $\pi$. The degree of the localization is controlled by the constant $\sigma$ and is the same at all radii. In a simple stellarator, the effect of allowing for $z_{0} \neq 0$ is to introduce a similar poloidal variation in the ripple amplitude as in a transport-optimised stellarator witt the wrong (negative) sign of $a$, but in this case the extent of the localization does vary with radius.

The upper limit in $y$ which must be included in the grid is determined principally by the highest $y$-value reached by particles in toroidally blocked orbits and hence by particles which detrap from ripple-trapped orbits. The code must use a mesh which extends to at least this point, and preferably some way beyond $i t$. The $a$-dependence of $E_{h}$ modifies this value.

According to Eq. (2), a particle which detraps on the inside of the device, at $\theta=\pi$, has $k / \mu B_{0}-1=\varepsilon_{h}(\theta=\pi)+\varepsilon_{t}$. Assuming that the minor radius of the particle is roughly constant, the maximum $y$ it attains, at $\theta=0$, is 


$$
y_{\max }=\left[2 \varepsilon_{t}+\varepsilon_{h}(\theta=\pi)\right] / 2 \varepsilon_{h}(\theta=0)+1 / 2
$$

If $\varepsilon_{h}=\hat{\varepsilon}_{h}(1-\sigma \cos \theta), y_{\max }=\left(1+\varepsilon_{t}\left(\hat{\varepsilon}_{h}\right) /(1-\sigma)\right.$. For positive vaiues of $\sigma$, for which the ripple is localised on the inside of the device, relatively larye values of $y$ must be included in the grid. Since $y \ll 1 / \varepsilon_{h}(r, \theta)$ is required for the assumptions made in the code to be valid, and $\varepsilon_{h}=\varepsilon_{h}(1-\sigma)$ at $\theta=0$, the condition is always satisfied by $y_{\max }$ on the outside of the device, and is satisfied at $\theta=\pi$ if $(1-a)$ is not too small.

For negative values of $\sigma$ (which may arise because the effects of $z_{0}$ being nonzaro are allowed for), the value of $y_{\text {max }}$ is decreased and so the lipper limit in $y$ of the grid may rorrespondingly be decreased.

\section{d) Results}

The diffusion coefficient for $\sigma= \pm 0,4$ is shown as a function of $y_{h} / \Omega_{E}$ in Fig. 2, superimposed on the analytic and numerical results for $\sigma=0$.

The radial electric field does not affect the mechanisn of the transport significantly in the $v^{-1}$ regime. The transport is mainly due to particles which are fatrly deeply trapped, in this regime, and th: benefit of having $a>0$, as may be seen from the equation for the radial drift velocity, $\dot{r}$, is that $\dot{r}$ is reduced for small values of $y$. Conversely, the disadvantage of $0<0$ is that $\dot{r}$ is increased for these same particles, which dominate the transport process. For particles which are barely trapped, however, the effect of $\sigma$ on $\dot{r}$ is reversed, so trat $\sigma>0$ leads to an increase in $\dot{r}$ for $0.83<y$, and $\sigma<0$ leads to a decrease. The antisymmetric nature of the perturbation to the distribution function in this regime is evident from fig. 7 (parts $2 a$ and $2 b$ ). In this respect the sulution is similar to that for $\sigma=$ 0 [4]. 
In the presence of a radial electric field and at low collision frequencies such that $v_{\text {eff }} \lesssim n_{\mathrm{E}}$, the transport in a standard stellarator is usually said to be dominated by the particles which are shallowly trapped [12], so that the $\sigma>0$ field might be expected [2] to have the more rapid transport and $\sigma<0$ the slower transport, the reverse situation to what is observed in the $v^{-1}$ regime. In fact, this is not what is found from the code, as: may be seen from Fig. 2. The $\sigma>0$ case nearly always shows significantly improved transport; $\sigma<0$ always shows more rapid (that is, worse) transport.

The key to understanding these ohservations may be the boundary-layer description of transport, for $\nu_{h} \lesssim \Omega_{E}$. For a true boundary layer, where $\Delta y_{b l}<1$, the relevant radial step, in the layer, would be largest for $\sigma>0$. However, $\dot{r}$ is only increased (for $\sigma>0$ ) when $y>0.83$. If the layer is all in the large $\dot{r}$ region above $y=0.83$, we must have $v_{h} / \Omega_{E} \lesssim 0.17^{2} \sim 3 \%$, which corresponds to a very low collision frequency, at which $D$ in the $v^{1 / 2}$ regime would be smat1. It is an interesting coincidence that the curve for $\sigma=0$ crosses that for $\sigma=0.4$ at almost exactly $v_{h} / \Omega_{\mathrm{E}}=0.03$, lending some support to this argument. As $v$ increases above this value, the thickness of the layer increases and it spreads into the region where $\dot{r}$ is small. Thus $D$ is insensitive to $v$, even before the boundary layer spreads to fill the rippletrapped region.

For $\sigma<0$, on the other hand, the particles which are in the layer but below $y=0.83$ have an increased $\dot{r}$, so as $v$ increases and the layer spreads, an increase in $D$ beyond that due to the increase in layer thickness (which is as $v^{1 / 2}$ ) occurs. Even when the width of the layer saturates, particles move to lower $y$ as $v$ increases and so $D$ continues to increase, al though somewhat more slowly. 
Figure $7(1 a, h)$ shows the distribution for $y_{h} / \Omega_{E}=0.12$. The contour density is seen to be much higher for $\sigma=-0.4$ than for $\sigma=0.4$, in both of the cases shown in Fig. 7 .

\section{Discussion and Conclusion}

A numerical method of solution of the bounce-averaged Fokker-Planck Eq., appropriate to stellarator transport at low collision frequency, has heen developed and used to study diffusion processes in the presence of a strong racial electric field in several stellarator configurations. A numerical approach to the problem was selected since analytic methods yield solutions which are limited in their applicability. In many situations it is unclear how an analytic calculation could be performed. In the few cases where such a calculation has been successful, the result is still not only restricted to certain special cases but in the $v^{1 / 2}$ and $1 / v$ regimes yields no information as to the distribution function in the non-ripple-trapped region. Particles which are not ripple-trapped have been shown in this study to have a significant effect on transport.

The scaling of the diffusion coefficient with collision frequency is of particular interest; in a typical case, $D$ varies as $v$ at the lowest callision frequencies, and as $v$ increases the variation of $D$ becomes as $v^{1 / 2}$, foltowed by what may constitute a separate region where $D$ is essentially independent of $v$. As $v$ increases still further, a decrease in $D$ as $v^{-1}$ is observed, although this is partially masked by the onset of the transport expected in an "equivalent" tokamak. In this region the numerical results conform closely to those obtained by summing the usual $v^{-1}$ result and the axisymmetric coefficient . 
Scaling of $D$ with other relevant parameters has been investigated in detail for the $v$ regime. Proviried we adopt the result of Ref. 16, which was derived for a similar problem in tokamak ripple transport, and and the two results given in Eqs. (5) and (6) reciprocally, the numerical resutts seem to confirm "theory."

In the $\nu^{1 / 2}$ regime and the plateau predicted for the saturated boundary layer diffusion, at $v_{h} \leq \Omega_{E}$, the diffusion coefficient agrees well with the predictions of the boundary-layer analysis of Galeev and Sagdeev, provided the integration over pitch-angle is performed correctly, as discussed above.

For "transport-optimised" stellarators, transport has heen studied at very low collision frequencies for the first time. The configurations having the better transport properties in the $v^{-1}$ regime also appear to have significantly better transport in the lower $v$ regimes than the standard stellarator. On the other hand, the type of configuration which shows more rapid transport in the $v^{-1}$ regime also has substantially worse transport for $v_{h} \lesssim \Omega_{E}$ than is usual.

The explanation of this is provided in part by the dependence of the radial drift velocity on pitch angle, $y$, in these configurations. For $v_{h}$ (the effective collision frequency) exceeding $\Omega_{E}$ (the $\vec{E} \times \vec{B}$ precession frequency), the transport is principally due to particles with small values of $y$. When $\nu_{h} \lesssim \Omega_{E}$, transport due to particles with $y \sim 1$ was expected to predominate, but this was not observed numerically. As discussed above, particles with $y<0.83$ still have a profound effect on transport for all but very low collision frequencies. Even at very low collision frequency, the idealisation usually made in analytic theory that the orbit shape is independent of $y$ is incorrect [11]. When this is allowed for, particles with $y<0.83$ will contribute for al 1 values. 
Comparison of these results with the results of Monte Carlo calculations must be done with care, since Tocal diffusion coefficients are difficult to obtain from Monte Carlo methods. If particles are followed for a long time, which is necessary in the low collision frequency regimes where a collision time is a substantial fraction of a second, many of those particles will escape from the region within which 0 is to be estimated. In this case, the way in which $D$ is calculated can significantly modify the results obtained. For instance, if $D$ is calcuiated from a dispersion formula $[11,14]$, a noticeable difference is obtained between the diffasian coefficients in the $v$ regime for $E_{r}>0$ and $E_{r}<0$. When a more sophisticated formula is employed which allows for an "absorbing boundary" [15], this difference is reduced. Nevertheless, some difference still appears to exist, in both the present and the Monte Carlo calculations, between the diffusion coefficients for apposite signs of the electric field. This we tentatively attribute to a second boundary layer, as discussed in Ref 11. (In this context we shouTd also point out that the difference between the diffusion coefficients was observed to decrease as $P_{t r}$ was decreased in Ref. 11. The unsatisfactory treatment of the trapping process in the framework of a purely bounce-averaged Monte Carlo calculation makes it difficult to discern whether a difference remains when $P_{t r}$ takes its true value in such a calculation. He remark again that this difficulty has been overcome by means of a "hybrid" bounceaveraged/guiding-centre calculation [15].)

The few approximations made in the present calculation seen to be justified, since, provided the trapping process is handled properly in the Monte Carlo codes, either by means of a full guiding-centre calculation, or by the use of guiding-centre Eq.s near $y=1$ in a 'nybrid' bounce-averaged/ guiding-centre calculation, the agreement between Monte Carlo results and the 
predictions of this code is very close indeed, at all collision frequencies and values of $\sigma$. In this context note that when $v$ is relatively large $\left(v_{h} \geq \Omega_{E}\right)$ it is necessary to allow for the full radial motion of particles in toroidally blocked orbits in order to obtain consistent results.

Finally, we would like to mention another, potentially important, development in the theoretical analysis of transport at low collision frequency in nonaxisymmetric devices such as stellarators. Hirshman [22] has developed a numerical means of evaluating the distribution function, without bounce-averaging. The method is effective for collision frequencies down to $v \leqslant w_{b}$, where $w_{b}$ is a typical bounce frequency, and so it is capable of treating some of the same processes as the present code. The approach of Hirshman has advantages and disadvantages as compared with that described in this paper, which we shall attempt to indicate.

The solution is sought on a flux surface by fourier analysing in the two angular variables and expanding the velocity space dependence in spherical harmonics. In order to restrict the calculation to a flux surface, the term involving the radial derivative of the perturbed distribution function is omitted. As discussed previously, this term is potentially important at low collision frequencies since although $\dot{r}$ is small, $\frac{\partial f}{\partial r}$ is not evaluated at constant $y$ in the calculation described in Ref. 22 and so $\dot{r} \frac{\partial f}{\partial r}$ is formally comparable to the other terms in the kinetic equation. Put another way, the variation in $y$ along the drift orbit of a ripple-trapped particie is a delicate balance between the effects of $r$ varying and $\theta$ varying. If the former variation is omitted in $\dot{y}$ [see Eq. (12)] the (de)trapping process, among other things, is not described correctly. For example, when this term is neglected, the particles will, for one sign of the electric field, appear 
to trap at the opposite side of the device from the true trapping point. Only by including the radial variation in the calculation of $\dot{y}$, as we have done, or some equivalent procedure, can this be rectified.

By the same token, however, Hirshman's code attempts to solve a more general problen than the present, bounce-averaged calculation. The problem with our approach may be summarised by saying that the simple bounce-averaging procedure employed here is not always appropriate. It is perhaps arguable whether it is more correct to restrict the calculation to a flux surface land avoid averaging) or to a set of surfaces which correspond to the bounceaveraged trajectories. For these reasons, we feel that the calculations are complementary. Where they agree (which, according to Ref, 22, they appear to do on the cases studied to date), we can assume that the results are correct; and where they disagree, it should be possible to see which assumptions are at fault, and so deduce the correct diffusion coefficient.

\section{Acknowledgment}

This work was supported by the 11.S. Department of Energy, under contract numbers DE-FGO2-85ER53201 and DE-AC02-76CH03073. 


\section{References}

[1] Mynick, H.E. and Hitchon, W.N.G., Nucl. Fusion 23 (1983) 1053.

[2] Kovrizhnykh, L.M., Nucl. Fusion 24 (1984) 851.

[3] Hastings, D.E., Phys. Fluids 28 (1985)334.

[4] Mynick, H.E. and Hitchon, W.N.G., Nucl. Fusion, in press.

[5] Goldston, R.J. and Towner, H.H., J. Plasina Phys, 26 (1981) 283.

[6] Martin, T.J., (private communication, 1978).

[7] Boozer, A.H, and Kuo-Petravic, G., Phys. Fiuids 24 (1981) 851.

[8] Wobig, H., Z. Naturforsch. 37a (1982) 906.

[9] Dommaschk, W., Lotz, W., and Nührenberg, J., Nucl. Fusion 24 (1984) 794.

[10] Wakatani, M., Mucl. Fusion 23 (1983) 817.

[11] Hitchon, W.N.G., Beidler, C.D., Mynick, H.E., and Shohet, J.L., Nucl. Fusion 25 (1985) 105. A bounce-averaged treatment of transport in EBT was presented in: J.S. Tolliver, Phys. Fluids 28 (1985) 1083.

[12] Galeev, A.A. and Sagdeev, R.Z., Reviews of Plasma Physics, 7, M.A. Leontovich, Ed., (Consultants Bureau, New York, 1979) 257 .

[13] Mynick, H.E., Phys. Fluids 26 (1983) 2609.

[14] Hitchon, W.N.G., Befdler, C.D., Mynick, H.E. and Shohet, J.L., J. Plasma Phys. 34 (1985) 327.

[15] Beidler, C.D., Hitchon, W.N.G., Mynick, H.E., and Shohet, J.L., Proc. Sherwood Theory Conference, (1985), Paper IS-18.

[16] Yushmanov, P.N., Dokladi Akademi Nayk CCCP 282 (1985)581.

[17] Hitshon, H.N.6. and Fielding, P.J., Nuct. Fusion 21 (1981) 775.

[18] Mynick, H.E., Chu, T.K., and Boozer, A.H., Phys. Rev, Lett. 45 (1980) 1328.

[19] Bykov, V.E., Georgievskij, A.V., Peletminskaya, V.G., Khodyachikh, A,V., and Shishkin, A.A., Nucl. Fision 24 (1984) 1195.

[20] Shaing, K.C., and Hokin, S.A., Phys. Fluids 26 (1983) 2136.

[21] Hitchon, W.N.G., McLenithan, K.D., and Mynick, H.E., Nucl. Fusion 23 (1983) 1143.

[22] Hirshman, S.P., Bur1. Am. Phys. Soc., (November, 1985), 513. 
TABLE 1

Field on Axis

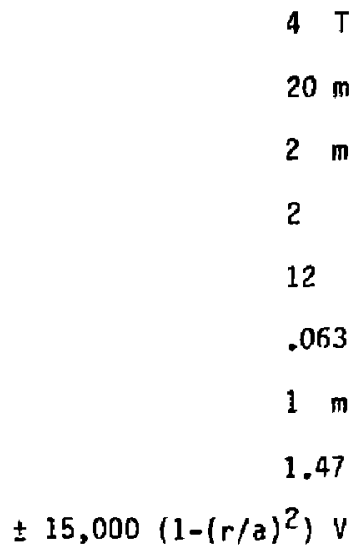

Major Radius

$0 \mathrm{~m}$

Minor Radius

Multipolarity (e)

2

$\varepsilon_{h}$ at Launch

.063

Launch Radius

$m$

4

$\pm 15,000\left(1-(r / a)^{2}\right) v$

Electrostatic Potential

$12 \mathrm{keV}$

Test Particle Energy

$10 \mathrm{keV}$

Background Energy 


\section{Figure Captions}

Figure 1 The geametry of the region where the solution is sought. Below $y=1$ only ripple-trapped particles exist, described by $f_{T}$. Above $y=1$ are toroidally trapped particles and passing particles, described by $f_{F}$ if they move forwards along a fietd line and $f_{B}$ if they move backwards. At $y=1$, toroidally trapped particles bounce or, with a small probability, $P_{t r}$, may become ripple trapped (on one side of the device only, in the Tatter case). Ripple-trapped particles detrap on reaching $y=1$, to become toroidally trapped.

Figure 2 Diffusion coefficient $D$ versus $v_{h} / \Omega_{E}$ for the device parameters given in Table I. The results for $\sigma=0.4,0$, and -0.4 are shown, as well as analytic predictions for $\sigma=0$, denoted by solid lines. The dashed line is the sum of the helical and the axisymetric diffusion coefficients $\left(O_{h}+D_{a}\right) \cdot D_{v r}$ is obtained from Eqs. (5) and (6), by adding them recipracally, whereas $D_{v}$ corresponds to Eq. (5).

Figure 3 The distribution function in the $v^{1 / 2}$ regime, obtained from analytic boundary layer analysis, 1) for a very narrow boundary layer, when $\left.v_{h} / n_{E}=0.05,2\right)$ for a layer of appreciable width, when $v_{h} / \Omega_{E}=0.15$ and 3) when $y_{h} / \Omega_{E} \approx 0.6$. In each case part a is from the analytic theory and $b$ is from the code. Note that despite the difference for $y>1$, the figures are very similar for $y<1$. In plots of the distribution the Maxwellian is represented by a value of 1 . Dotted lines indicate the total distribution is less than one and solid lines the distribution is greater than one. The contour spacing is 0.005 . 
Figure 4 D versus $\varepsilon_{h}$ in the $v^{1 / 2}$ regime.

Figure 5 D versus $E_{h}$ in the $v$ regime.

Figure 60 versus electric field strength in the $v$ regime, the $v^{1 / 2}$ regime and the "saturated" boundary layer regime, where $D$ is independent of $v$. The scaling with $E_{r}$ is in excellent agreement with "theory," the slopes being $-2,-3 / 2$, and -1 in the three cases, respectively. $E_{r}=\alpha E_{r o}$, where $E_{r o}$ is the value indicated in Table I.

Figure 7 The distribution function for $v_{h} / \Omega_{E}=0.12$ and 1.2 , in the top and battom rows, respectively. In each case, the left figure is for $\sigma=$ 0.4 , the right figure is for $\sigma=-0.4$. 


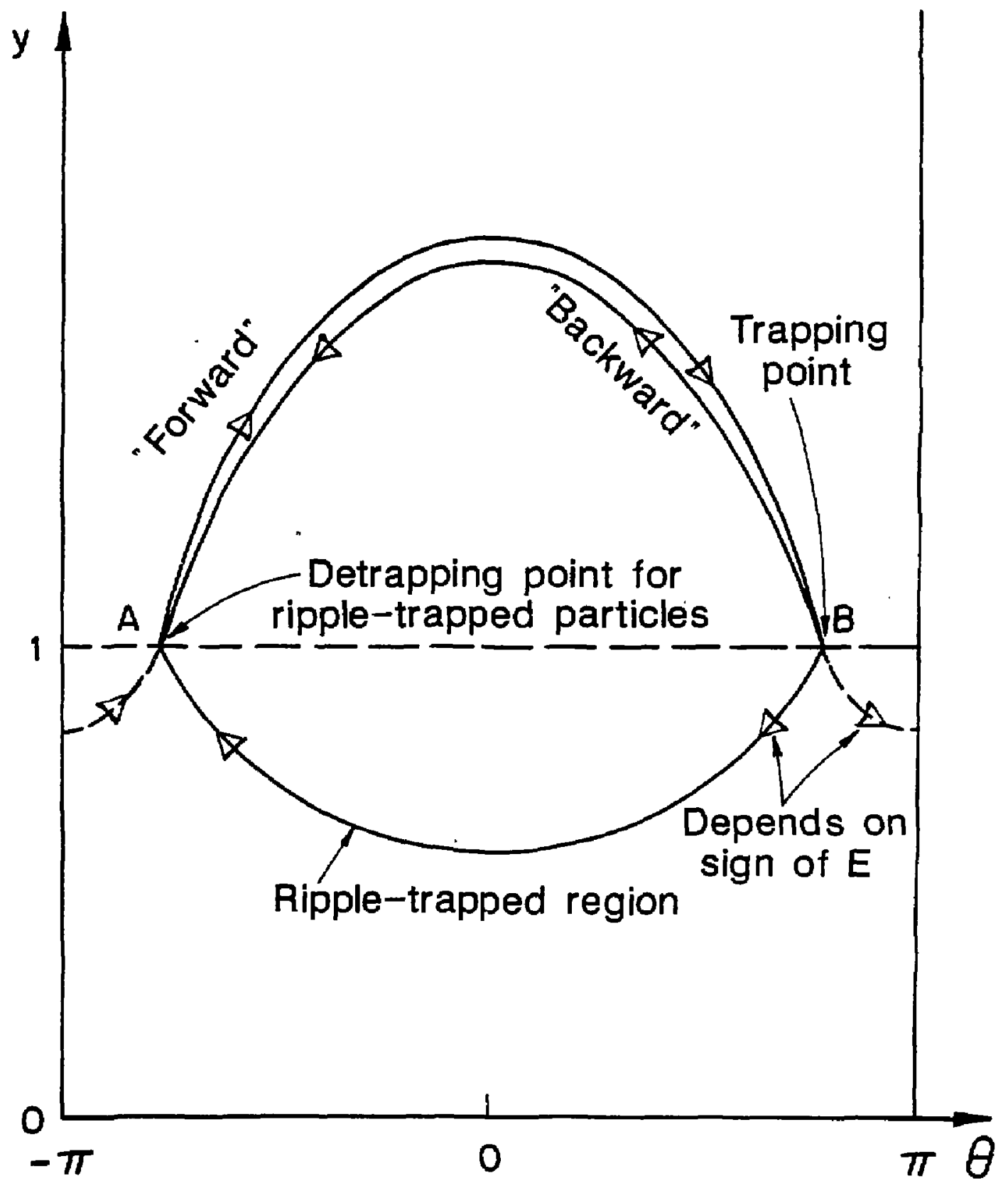

Fig: 1 


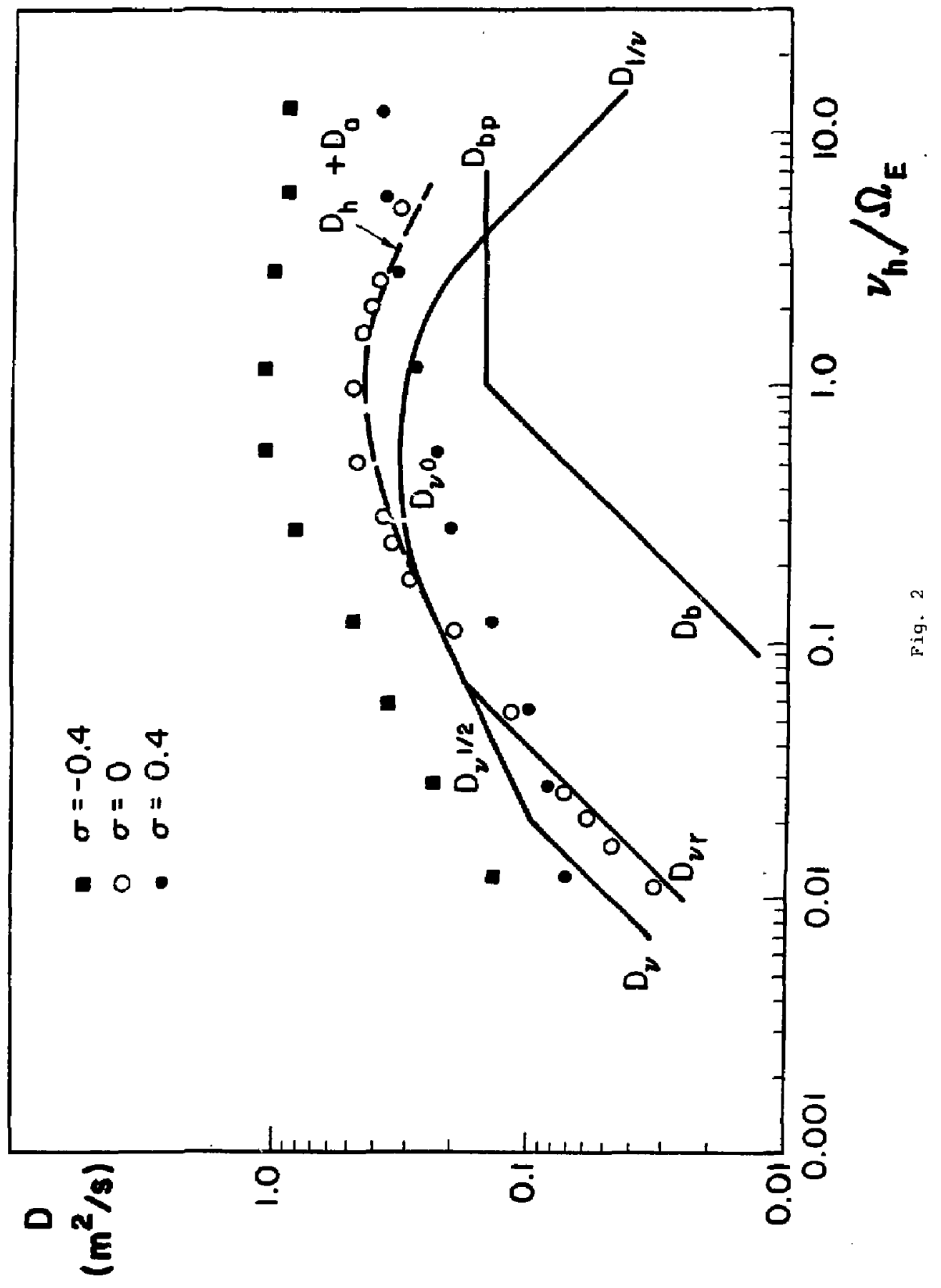



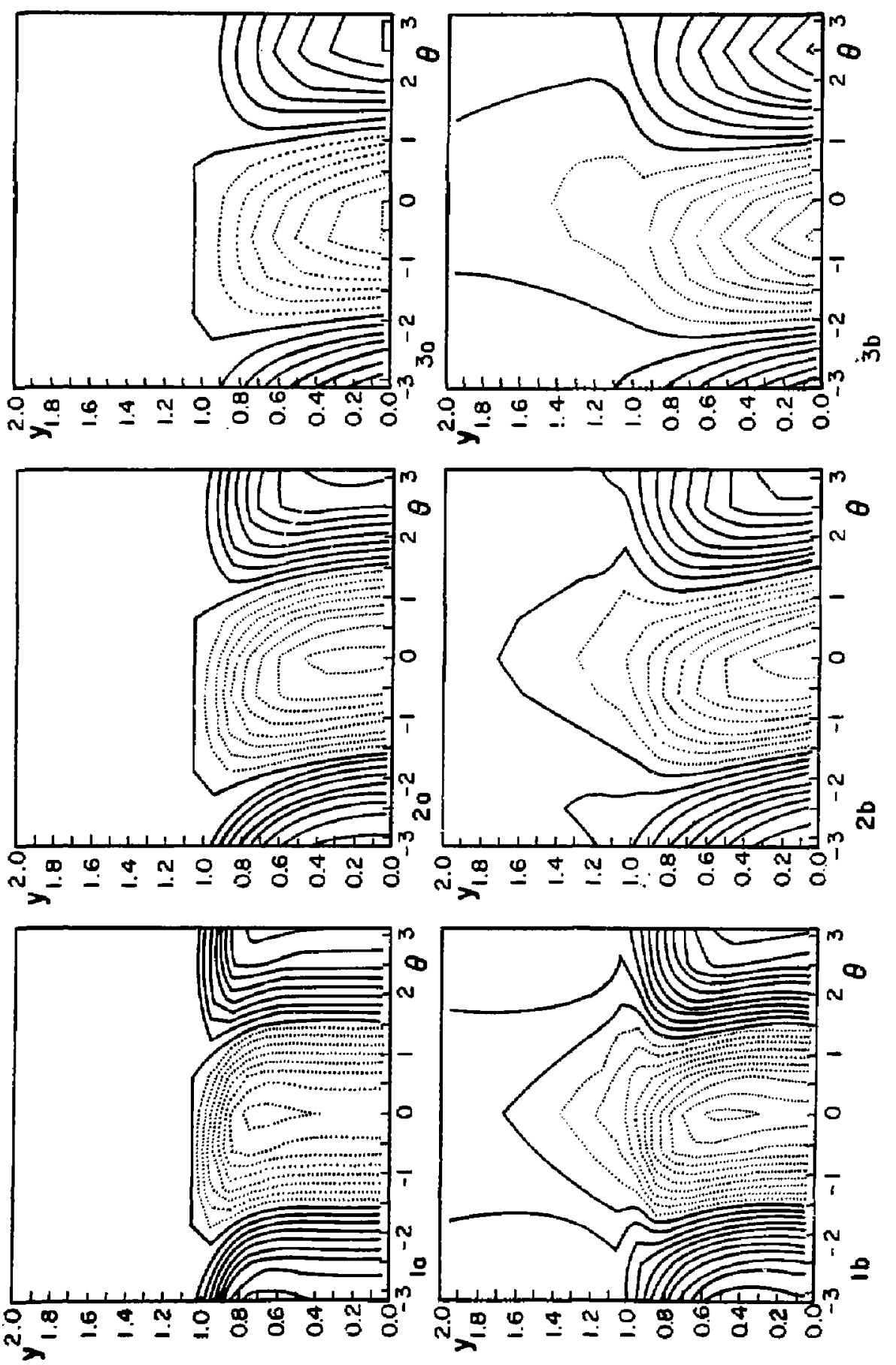

它 


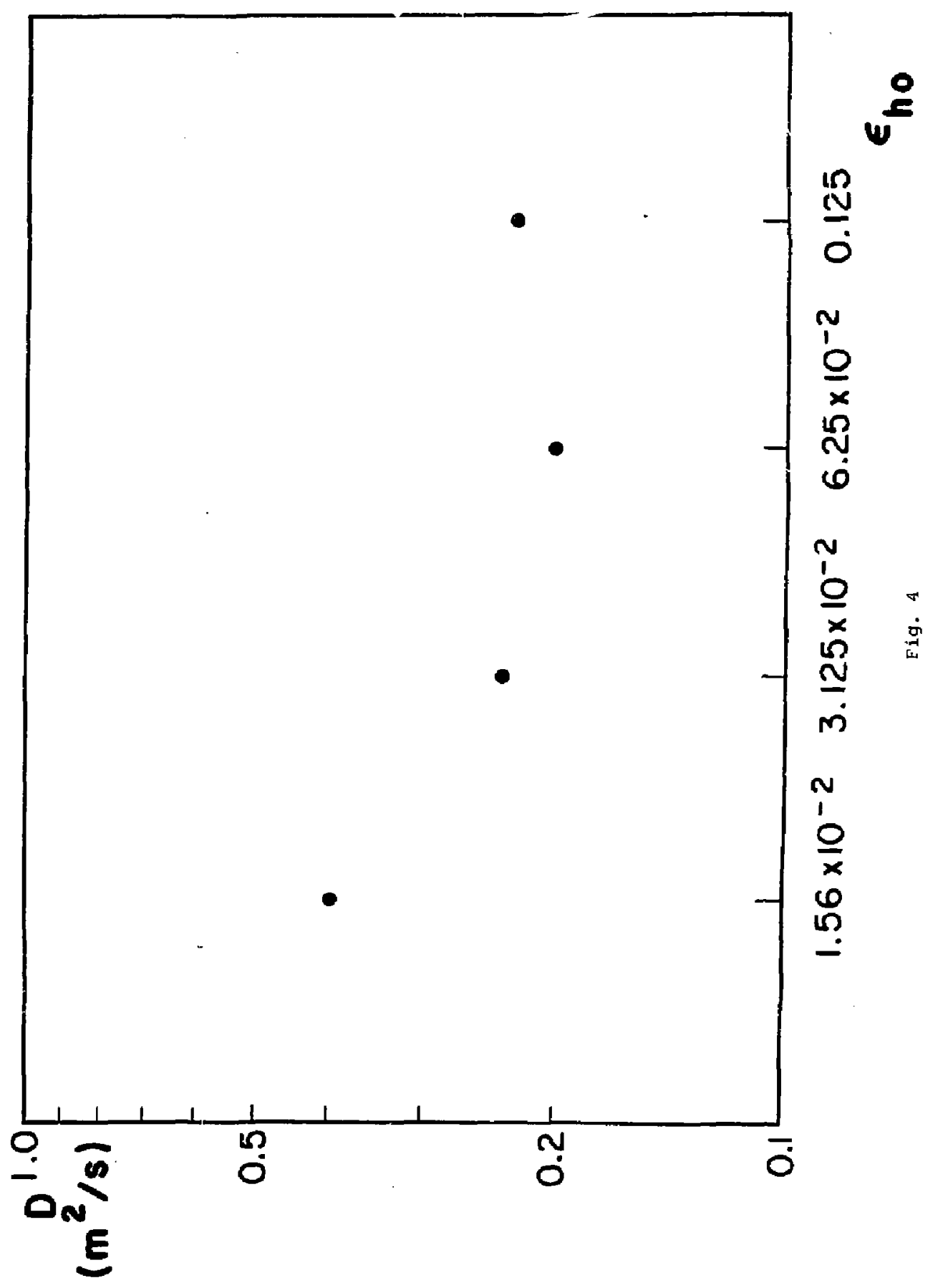




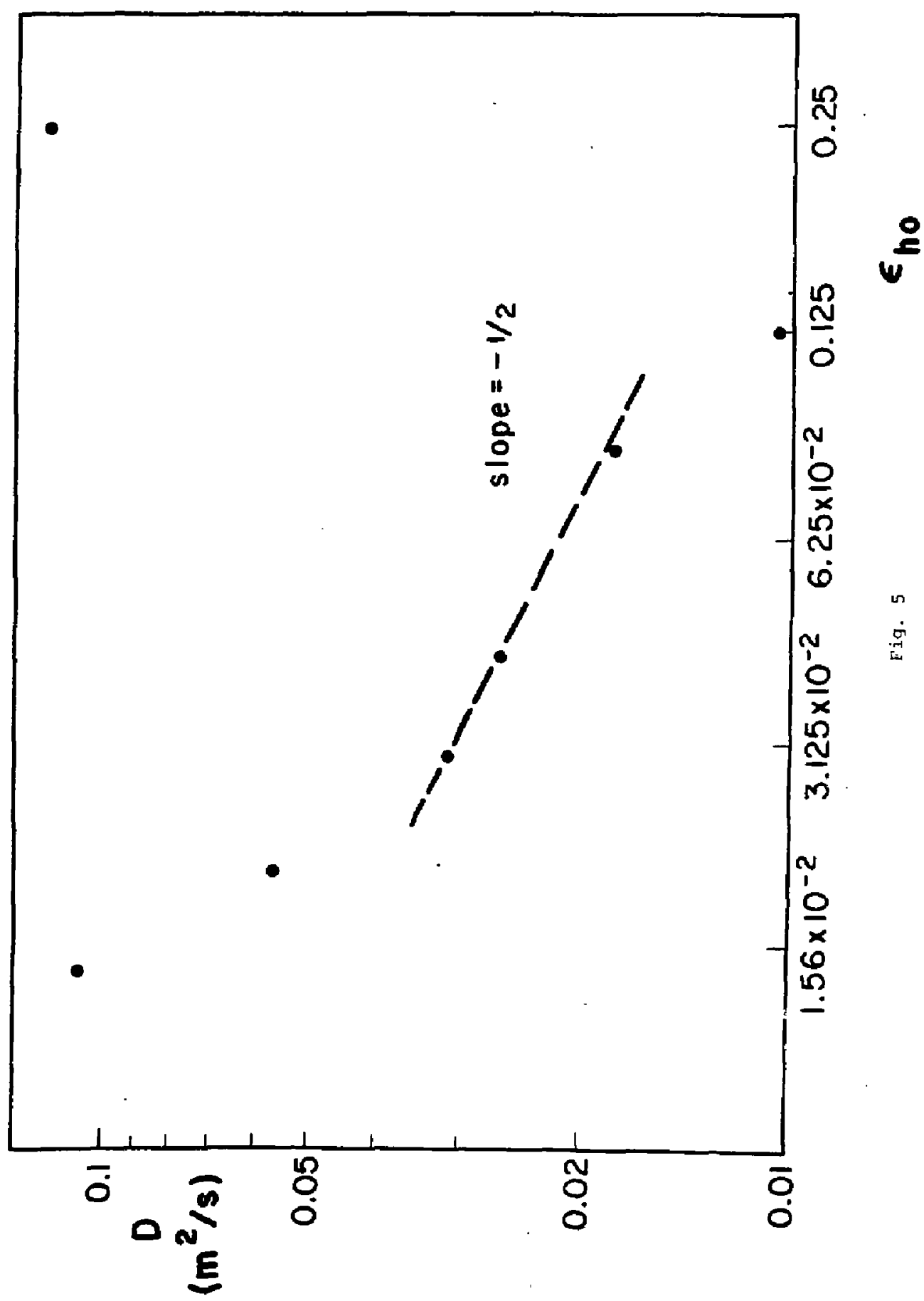




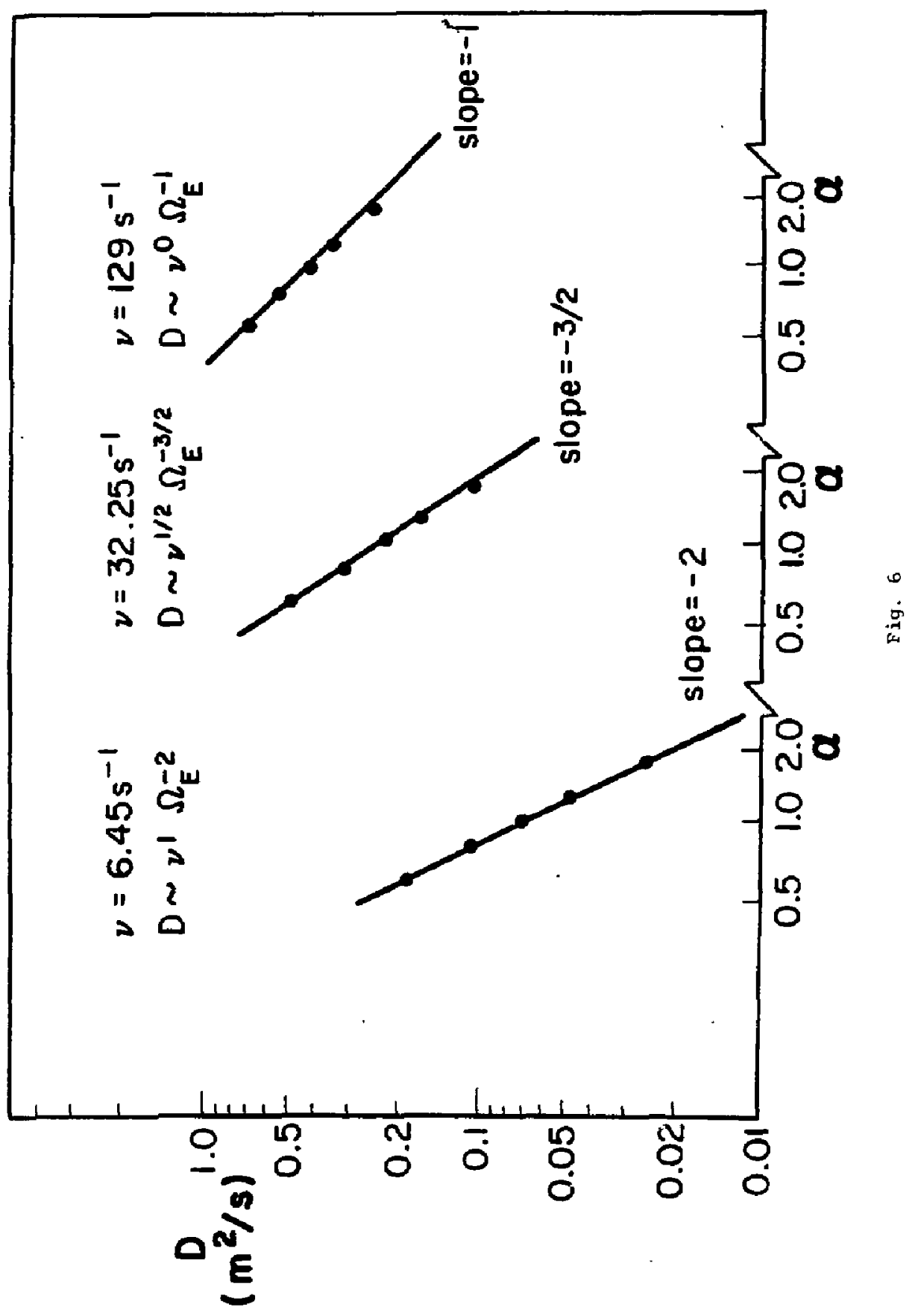



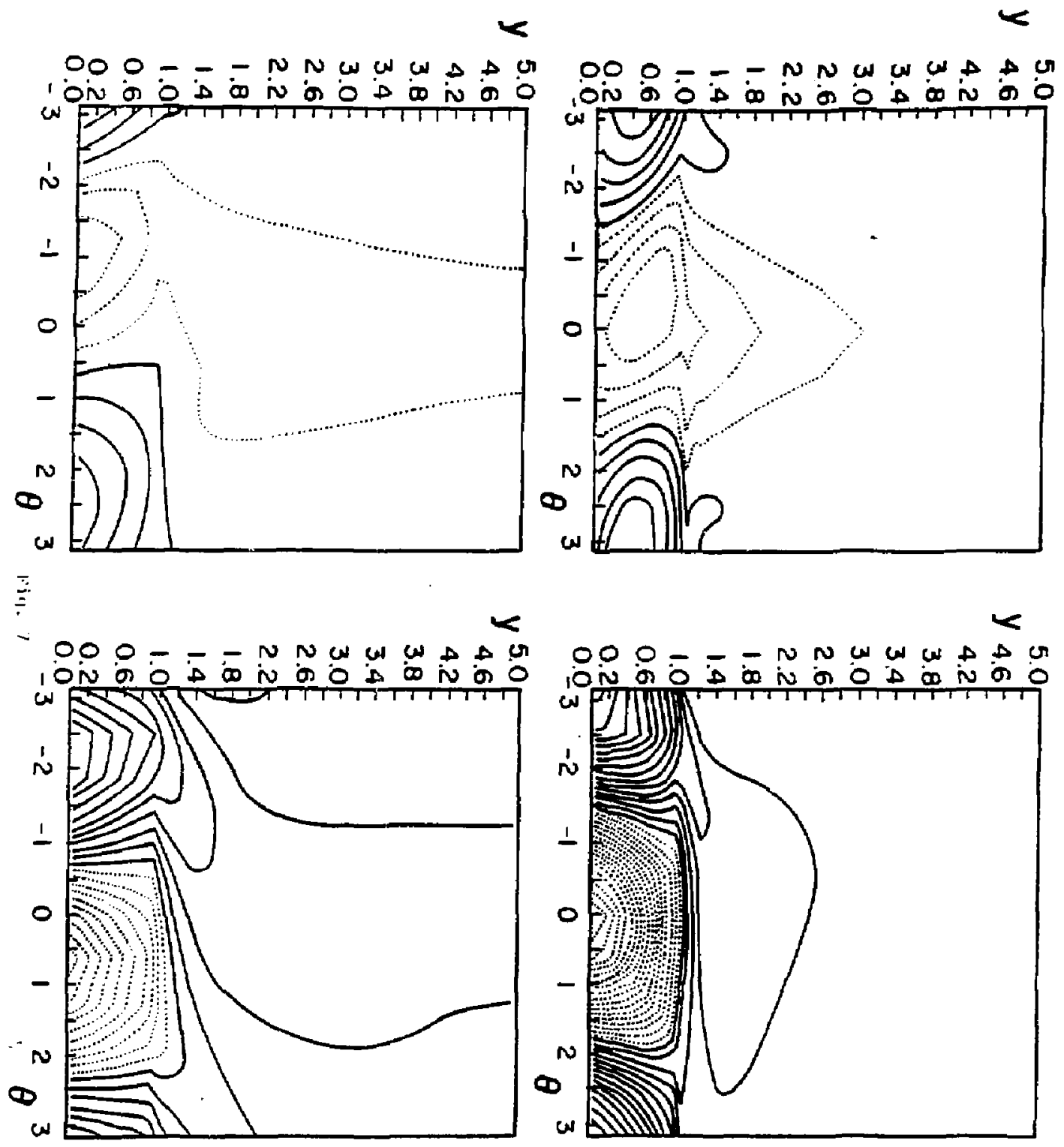
Plasme Res Lab, Austra Nat'i UnIv, aUSTRalia

Dr. Frank J. Paoton 1, Univ of wallongong, AUSTRALIA

Pre? I,R. Jones, Fi inders Univ.., AuSTRALIA

Prof. M.H. Brennan, UnIv Sydney, Australla

Prof. F. Cap, Inst Theo Phys, AuSTR1A

M. Goossens, Astronomlsch Instituut, BeLGIUM

Prof. R. Bouclqu*, Laborator lum roor Natuurkunde, GELGIuM

Dr. D. Palundo, Dg XII Fusion Prog, Belgium

Ecole Royale Militafre, Lab do Phys Plasmas, GELGicha

Dr. P.H. Sakanaka, Univ Estadual, BRNIL

LIb. I Do. DIv., Instituto do Pesquisas Espaclala, BRNIL

Or. C.R. James, Univ of Alberta, CANNOA

Prof. J. Telehmann, Univ of Montreal, GANADA

Dr. H.M. Skarsgard, Univ of Saskotcheman, CANADA

Prof. S.R. Srgenivosan, Universlty of Calgary, CAHAOA

Prof. Tudar W. Johnston, INRS-Energle, CANADA

Dr. Hennes Barnard, Unlv Brittsh Columbla, CANABA

Dr. M.P. Bachynskl, Bechnologles, Inc., CANADA

Chalk RIver, Nuct Leb, CANADA

Znengwa LI, SH Inst Physics, CHINA

LIbrary, Tsling Hua Unlverstity. CHINA

Liorarlan, Instifute of Physles, CHIMA

1nst Plasnd Phys, Acodomlo Sinico, CHINA

Dr. Peter Lukac, Komenskaho Un1r, CechosLOYAKiA

The Librarlan, Culham Laboratory. ENELAND

Prof. Schatzman, Observatolre de NICe, FRANCE

J. Radet, CEN-BP6, FRAMCE

JET RaAding Room, JET Joint Undertaking, ENGLAND

M Dupas Library, MH Dupas LIbrary, FRAMCE

Dr. Ton mual, Academy Blbllographic, HONG KONG

Preprint LIbrary, Cent Res Inst Phys, HUNGMay

Dr. R.K. Chhajlanl, WIkram Univ, INDIA

Dr. B. Oasgupta, Saha Inst, IROIA

Dr. P. Kas, Physical Rasaarch Lab, INDIA

Dr. PhIIllp Rosenau, Israel Inst Tech, ISRAEL

Prot. 5. Cuporman, Tol Aviv Universlty. ISRAEL

Prof, G. Rostogn1. Uniy DI Padovs, ITALY

LItrarlan, Int'I Ctr Theo Rhys, ITALY

MIss Clella De Palo, Assoc ElAATOM-ENEA, ITALY

Blbllotoca, dol CNR ELAATOH, ITALY

Dr. H. Yamato, Toshlba Res \& Dov, JAPAN

Dlrec, Dept, Lg. Tokanak Dev, JAEAI, JAPAN

Prof. Nobuyuk! Inoua, Univorsity of Tokyo, JAPAN

Research Into Center, Nagoya University, JAPAN

Prof. KyoJl NIshIkawa, UnI of HIroshlma, JAPAN

Praf, SIgaru Morl, JAERI, JAPAH

Prof. 5. Tanaka, Kyoto Unlverslty, JAanN

LIbrary, Kyoto Unlyersity, JAPAN

Prot. Ichiro Kawakeml, Nihon Unit, JAPAN

Prof, Sotoshi Itoh, Kyushu University, JAPAN

Dr. D.1. Chol, Adr. Inst Scl \& Tech, KOREA

Tuch Info DIvIsion, KAERI, KOREA alollotheak, Fom-Inst Yoor Plasma, NETHERLANDS Prof. B.S. LII ay, UnIversity of Walkato, NEW ZEALAND Prof. J.A.C. Cebrol, Inst Suparior Tocn, PORTUGAL Dr. Oetavian Patrus, ALI CURA University, ROHANIA Prot. M.A. Hellberg, Unlverslty of Natal, SO AFRICA Dr. Johen de VItllers, Plasna Physles, Nucor, SO AfRICA Fuslon Div. LIbrary, JEN, SPAIN Prot. Hans Wlihoimsan, Chalmers UnIr Toeh, SHEDEN Dr. Lonnart Stenfla, UnIversity of IMEA, SWEDEN LIbrary, Royal Inst Toch, SWEDEN

Centre de Recherchesen, Ecole Polytech Fed, SixITERLAND Dr. V,T. Tolok, Kherkov Phys Tech InS, USSR

Dr. D.D. P.yutor, SI ber I an Acad SEI, USSR

Dr. G.A. EIIseer, Kurchator Institute, USSR

Dr. Y.A. Glukhlkh, Inst Electro-Physlcal, USSR Institute Gen. Physlcs, USSR

Prot. T.J.M. Boyd, UnIv Colloge N Wales, WALES Dr, K, Schindler, Ruhr Universitot, $N$, GERMANY ASDEX Reading Rn, IPP Max-PIanck-institut fur

Plasmephyslk, F,R,G.

Nuc loar Ros Estab, Jul Ich L+d, W. GERMANY Elorarlan, Hox-Planck Instltut, W. GERMANY Bibllothok, Inst Plasmotorschung, W, GERAMNY Prot, R,K, Jonov, Inst Phys, YugosLavia 